\title{
The effects of qualitative and quantitative variation in the US on individual components of Pavlovian appetitive conditioned behavior in rats
}

\author{
PETER C. HOLLAND \\ University of Pittsburgh, Pittsburgh, Pennsylvania 15260
}

\begin{abstract}
The form of rats' Pavlovian conditioned responses to visual and auditory conditioned stimuli (CSs) paired with a variety of unconditioned stimuli (USs) was examined in three experiments using direct behavioral observation techniques. In Experiment 1, the form of conditioned behavior occurring most frequently during later portions of the CS-US interval depended only on which of several appetitive USs was used, but the form of behavior occurring most frequently during early portions of the CS-US interval depended only on the nature of the CS. US-dependent behaviors resembled the response to the US, and CS-dependent behaviors resembled the original orienting response (OR) to the CS. In Experiment 2, the use of larger magnitude appetitive USs resulted in higher frequencies of US-dependent behaviors, but lower frequencies of CS-dependent behaviors in the presence of auditory and visual CSs. In Experiment 3, US-dependent conditioned behavior to auditory and visual CSs paired with shock was more frequent when high-intensity shocks were used, but CS-dependent behavior was more frequent when low-intensity shocks were used. These results suggested that Pavlovian conditioned responding may involve two independent types of behavior-one appropriate to the US and another based on the original OR to the CS.
\end{abstract}

The form of the Pavlovian conditioned response (CR) has usually been considered to be dependent chiefly on the nature of the unconditioned stimulus (US) or response (UR) event (e.g., Hilgard \& Marquis, 1940; Jenkins \& Moore, 1973; Rescorla \& Solomon, 1967). Recently, however, Holland (1977) has shown that very gross features of CR form may be equally dependent on the nature of the conditioned stimulus (CS).

Using direct behavioral observation techniques, Holland (1977) found that rats' behavior in the presence of CSs preceding food delivery differed greatly depending on the modality of the CS. Responding to visual CSs consisted mainly of two behaviors, rearing and magazine. Auditory CSs, however, evoked first a startle response to CS onset, followed by head-jerk behavior; lesser, but significant, amounts of magazine behavior also occurred, especially during the early sessions of conditioning. The behaviors evoked by auditory and visual CSs differed sufficiently to generate substantial differences even in a crude stabilimeter measure of general activity. Auditory CSs came to evoke large amounts of general activity,

This research was supported in part by Grants 5207RR0708-11 and MH31396-01 from the National Institutes of Health. Requests for reprints should be sent to Peter Holland, Department of Psychology, University of Pittsburgh, Pittsburgh, Pennsylvania 15260 . but visual CSs did not acquire the ability to evoke activity.

Holland (1977) suggested that the complex CRs that he observed might reflect the interaction of two quite independent sources of responding. One component of the CR might be determined by the US, while another component might be a result of conditioning-dependent enhancement of a previously unconditioned orienting response (OR) to the particular CS involved. Several aspects of Holland's data supported this claim. First, behavior occurring during the later portions of the CS-US interval was directed towards the food magazine with both auditory and visual CSs; the one behavior common to both CSs, magazine, was similar to the behavior evoked by food delivery itself. Second, the ordinal amounts of rearing and startle behaviors to a variety of stimuli were predictable from the ORs evoked by those stimuli on their initial presentation prior to conditioning; further, in conditioning, these CSspecific behaviors were largely confined to early portions of the CS-US interval, when an OR would be expected to occur.

This "orienting response" hypothesis implies that variations in CS quality would affect "CS-determined" behaviors occurring early in the CS interval but leave "US-determined" behavior occurring later in the interval relatively intact, and that variation in US quality would affect late-CS interval behavior but 
have lesser effects on early-CS behaviors such as rearing and startle. Elsewhere, Holland (in press) has provided support for the first implication; the experiments reported here examine the nature of responding to auditory and visual CSs paired with a variety of qualitatively different appetitive USs. Experiment 2 examines responding to CSs paired with quantitatively different food USs. Experiment 3 examines responding to CSs paired with differentintensity aversive (shock) USs.

\section{EXPERIMENT 1}

Experiment 1 examined responding to a noise and a light CS paired with one of three appetitive US events: food pellets delivered to a recessed magazine, food pellets delivered to a protruding cup, and sucrose solution delivered to a cup. Each rat received only one type of US and only one type of CS paired with that US, but also received the other type of CS nonreinforced.

\section{Method}

Subjects and Apparatus. The subjects were 36 male SpragueDawley rats about 100 days old at the start of the experiment. They were maintained at $80 \%$ of their normal body weights throughout the experiment, and lived in a constantly illuminated colony room.

Four experimental chambers, each $22.9 \times 20.3 \times 20.3 \mathrm{~cm}$, were used. Each chamber had a dimly illuminated, recessed food magazine in the center of one end wall and a $4 \times 3 \times 2.5 \mathrm{~cm}$ food cup protruding from that same wall, $2 \mathrm{~cm}$ to the left of the food magazine. The floor was composed of .48 -cm stainless steel rods spaced $1.9 \mathrm{~cm}$ apart. The two end walls were aluminum; the side walls and top were clear acrylic plastic. Each chamber was enclosed in a sound-resistant shell. A speaker for delivering the noise CS and a 6-W lamp used as the visual CS were mounted on the wall of the shell, directly behind the center of one side wall of the experimental chamber. Each sound-resistant shell had a transparent acrylic window in one side to permit behavioral observations; the shells were located in a soundproof room.

The television system for observing and recording the rats' behavior included a low-light-level camera mounted $2.1 \mathrm{~m}$ from the experimental chambers so as to include all four chambers in its field of view, a video recorder, and a 19-in. monitor. The recorder was programmed to record behaviors for $10 \mathrm{sec}$ before, $10 \mathrm{sec}$ during, and $10 \mathrm{sec}$ after each CS presentation. The video recorder and all other programming equipment were located in a room separate from the experimental chambers.

Observation procedure. All observations were made from video tapes. The observer shifted his gaze from chamber to chamber so as to observe each of the four rats twice during each $10-\mathrm{sec}$ pre-CS interval and twice during each CS interval-once during the first half of the interval, and once during the last half. A total of eight observations, two per rat, were made during each 10-sec pre-CS or CS interval. In addition, the experimenter made one observation of each rat during the first $5 \mathrm{sec}$ after each US presentation during conditioning. Observations were paced by a recorded auditory signal. This procedure yielded the measure "percent total behavior," obtained by dividing the number of occurrences of each behavior by the total number of observations made. Note that this is an absolute measure, not a relative one, since the number of observations is constant. The data are expressed as percentages of total observations rather than as abso- lute frequencies to facilitate comparisons with data of other experiments (e.g., Holland, 1977, 1979) in which the total number of observations made differed from that made in these experiments.

Behavioral categories. Six categories of behavior are reported in these experiments. (1) Startle, a sudden rapid body movement resulting in a change in position, usually occurring within $1.25 \mathrm{sec}$ of CS or US presentation (before the first pacing signal recorded on the video tapes); the movement was usually, but not necessarily, directed towards the reinforcement site. (2) Magazine, standing motionless in front of the food magazine with nose or head within the magazine, (3) Head jerk, short, rapid, horizontal, and/or vertical head movements, usually, but not necessarily, directed towards the reinforcement site; they might occur with paws on cup or head partially in magazine. (4) Rear, standing on hindlegs with both front feet off the grid floor, unless the rat was grooming (see below). (5) Groom, rubbing forepaw over any part of head, or contacting body, hindlegs, or tail with forepaws or mouth. (6) Cup, standing motionless with front paws on the food cup, back arched, and nose or head in the food cup.

The first five categories have been used extensively in previous research; more complete descriptions of these behaviors can be found in Holland (1977). Cup was developed from extensive preliminary examination of the video tapes of rats' behavior in Experiment 1. Another behavior, quiet, consisting of a number of activities, such as sniffing, sleeping, and gnawing on grid bars, was recorded but is not reported here as it did not occur to any significant extent to conditioned or unconuitioned stimulus presentation. These categories have been shown to be easily and reliably judged among several observers in other studies (e.g., Holland, 1977, 1979).

Procedure. In the first session, rats were randomly assigned to one of six groups of six rats each and trained to approach and consume either $45-\mathrm{mg}$ Noyes pellets or $16 \%$ sucrose solution in the experimental chamber. Rats in Groups $\mathrm{Nfc}$ and $\mathrm{Lfc}$ received eight deliveries of one food pellet into the food cup over a period of $10 \mathrm{~min}$; rats in Groups $\mathrm{Nfm}$ and $\mathrm{Lfm}$ received eight deliveries of one food pellet into the recessed food magazine; and rats in Groups Nsc and Lsc received eight deliveries of 1 cc sucrose solution into the food cup.

The next session served as a pretest of the stimuli to be used in conditioning. Each rat received four $10-\mathrm{sec}$ presentations of a $75-\mathrm{dB}$ (A scale, re $20 \mathrm{~N} / \mathrm{m}^{2}$ ) white-noise CS and four 10 -sec presentations of the light CS. This session and those of the next phase were each $75 \mathrm{~min}$ in duration; no stimuli were delivered during the first $15 \mathrm{~min}$ of any session. Intertrial intervals averaged $7.4 \mathrm{~min}$ throughout the experiment.

Each rat then received eight conditioning sessions. In each session, rats in Group $\mathrm{Nfc}$ received four presentations of the 10-sec noise CS followed immediately by delivery of food into the food cup and four nonreinforced presentations of the light stimulus; rats in Group $L f c$ received four light-food pairings and four nonreinforced noise trials; rats in Group $\mathrm{Nfm}$ received four pairings of the noise with food delivered to the food magazine and four nonreinforced light trials; rats in Group Lfm received four pairings of the light with food delivered to the food magazine and four nonreinforced noise presentations; rats in Group Nsc received four noise-sucrose pairings and four nonreinforced light presentations; and Group Lsc received four light-sucrose pairings and four noise-alone trials.

Experiment 1 was conducted in two replications. There were no differences between the replications.

\section{Results}

Pre-CS behavior. About $90 \%$ of the pre-CS behavior in all groups over the entire conditioning phase was quiet behavior. The remaining $10 \%$ of pre-CS behavior consisted of rear, cup, and magazine. Separate analyses of variance indicated that none of these 
Table 1

Responding to the US in Experiment 1

\begin{tabular}{lcrrr}
\hline & \multicolumn{4}{c}{ Behavior } \\
\cline { 2 - 5 } Group & Startle & Magazine & \multicolumn{1}{c}{ Cup } & Groom \\
\hline Nfm & 85.0 & 95.8 & .7 & 2.1 \\
Nfc & 78.3 & 1.4 & 95.8 & 1.4 \\
Nsc & 77.5 & .7 & 84.7 & 13.9 \\
Lfm & 85.8 & 96.5 & .7 & 1.4 \\
Lfc & 79.2 & .7 & 95.8 & 1.4 \\
Lsc & 84.2 & .0 & 81.2 & 13.9 \\
\hline
\end{tabular}

Note-Data for startle responding are expressed in percentage of trials on which a startle occurred. Remaining data are expressed as percentages of total behavior.

behaviors differed significantly in frequency between groups $[\mathrm{Fs}(5,30)<1$, ps $>.10]$.

Response to US delivery. Feeder operation usually elicited a startle response, approach to the food tray or cup, and finally, magazine, cup, or grooming behavior. Table 1 shows the frequency of startle, magazine, cup, and grooming behavior over the eight conditioning sessions. US Type by CS Type analyses of variance showed that the frequencies of magazine $[\mathrm{F}(2,30)=2,644.34, \mathrm{p}<.01]$ and cup $[\mathrm{F}(2,30)=$ $200.12, \mathrm{p}<.01]$ behaviors were affected by the type of US used. Individual comparisons using the Scheffé procedure showed that Groups Nfm and Lfm each showed more magazine and less cup behavior than any of the other groups $[\mathrm{Fs}(5,30) \geqslant 112.38$, ps $<.01]$. Neither of these behaviors was affected by the nature of the CS (noise or light) which preceded US delivery (Fs $<1$ ). Grooming differed depending on US type $[F(2,30)=52.11, p<.01] ;$ Groups Lsc and Nsc (which received the viscous sucrose solution) each showed more grooming than any of the other groups $[\mathrm{Fs}(5,30) \geqslant 35.83]$. The occurrence of post-US grooming was unaffected by the type of CS which preceded US delivery $[F(1,30)<1]$. Startle responding to US delivery was not affected by either US type or CS type (Fs $<1)$.

It should be noted that the behavior evoked by US delivery should not be labeled "unconditioned" responses, for prior to magazine training, the audible feeder clicks did not evoke approach to the food tray or cup. Although feeder clicks did initially evoke startle responding, other data from this laboratory indicate that if the click is not followed by food consumption, startle responding is not maintained. Thus, startle behavior to US delivery was probably a conditioned response to the auditory feeder click (see below).

Behavior to noise CS. The four left frames of Figure 1 show behavior evoked by the noise stimulus in all six groups over the course of conditioning. A US Type by Contingency (noise paired or unpaired with the US) analysis of variance over the eight conditioning sessions showed that the frequency of startle responding to the noise was unaffected by US type $[F(2,30)<1]$; the occurrence of startle responding to the noise was, however, dependent on noise-US pairings $[F(1,30)=318.31, \mathrm{p}<.01]$. Similarly, although the occurrence of head-jerk behavior to the noise was dependent on noise-US pairings $[F(1,30)=391.94, p<.01]$, it was unaffected by US type $(\mathrm{F}<1)$.

Conversely, the frequency of magazine behavior to the noise was affected by US type $[F(2,30)=33.36$, $\mathrm{p}<.01$ ]; that behavior was more frequent in Group Nfm than in Group Nfc or Group Nsc [Scheffé Fs $(5,30) \geqslant 72.3$, ps $<.01]$. Only Group Nfm differed in magazine frequency from its corresponding control, Group Lfm, in which food delivered to the food tray was paired with the light but not the noise $[F(5,30)=65.04, p<.01]$. Similarly, the frequency of cup behavior to the noise depended on US type $[\mathrm{F}(2,30)=31.17, \mathrm{p}<.01]$; cup behavior was more frequent in Groups $\mathrm{Nfc}$ and Nsc than in Group $\mathrm{Nfm}$
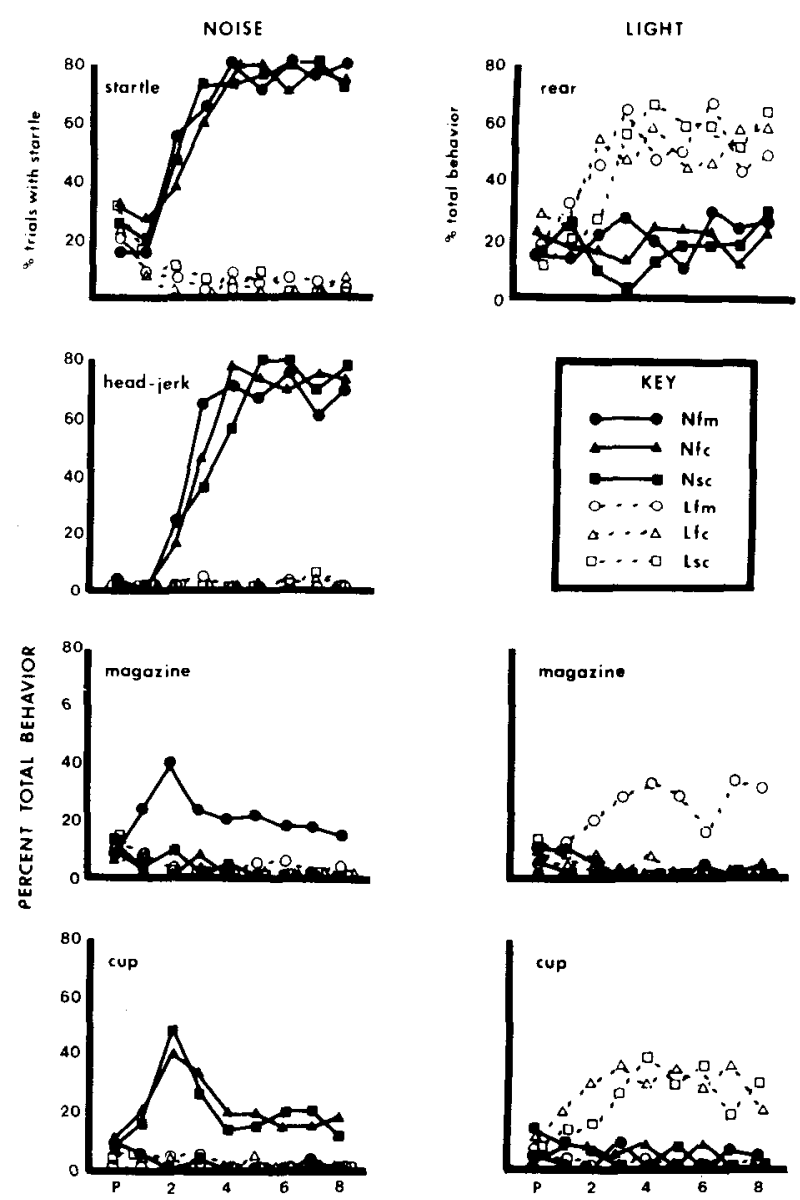

SESSIONS

Figure 1. Behaviors to noise and light stimuli during pretesting and conditioning in all six groups of Experiment 1. Frequency of each behavior, except startle, is expressed as a percentage of total behavior; startle behavior is expressed as the percentage of trials on which a startle occurred. 
$[\mathrm{Fs}(5,30) \geqslant 88.68$, ps $<.01]$. Only Groups Nfc and Nsc differed from their corresponding controls, Groups Lfc and Lsc, respectively $[\mathrm{Fs}(5,30) \geqslant 59.10$, $\mathrm{p}<.01]$. Cup behavior did not differ significantly between Groups $\mathrm{Nfc}$ and $\mathrm{Nsc}(\mathrm{F}<1)$.

Neither rearing nor grooming behaviors occurred in the presence of the noise in any of the groups in which that CS was paired with a US.

Behavior to light CS. The right three frames of Figure 1 show behavior evoked by the light stimulus in all six groups over the course of conditioning. The frequency of rearing to the light was unaffected by US type $[F(2,30)<1$, ps $>.10]$, but was dependent on light-US pairing $[\mathrm{F}(1,30)=51.45, \mathrm{p}<.01]$.

Conversely, the frequency of magazine behavior to the light was affected by the type of US used $[\mathrm{F}(2,30)=20.55, \mathrm{p}<.01]$; that behavior was more frequent in Group Lfm than in Group Lfc or Group Lsc $[\mathrm{Fs}(5,30) \geqslant 70.54$, ps $<.01]$. Only Group Lfm differed in frequency of magazine behavior from its corresponding control, Group $\operatorname{Nfm}[\mathrm{F}(5,30)=83.34$, $\mathrm{p}<.01$ ]. Similarly, the frequency of cup behavior to the light CS was dependent on US type $[\mathrm{F}(2,30)=$ 43.04, $p<.01$ ]; cup behavior was more frequent in Groups Lfc and Lsc than in Group $\operatorname{Lfm}[\mathrm{Fs}(5,30)$ $\geqslant 100.67$, ps $<.01]$. Only Groups Lfc and Lsc differed from their corresponding controls (Groups Nfc and Nsc, respectively) in the frequency of cup behavior $[\mathrm{Fs}(5,30) \geqslant 88.07$, ps $<.01]$. Cup behavior to the light $\mathrm{CS}$ did not differ significantly between groups Lfc and $\operatorname{Lsc}(F<1)$.

Neither grooming, head-jerk, nor startle behaviors occurred in the presence of the light CS.

Temporal distribution of $\mathrm{CS}$ behaviors. Table 2 shows the frequencies of behaviors during the first and last 5-sec periods of the noise and light CSs over all conditioning sessions. Those behaviors which were unaffected by type of US were those occurring more frequently during the first half of the CS interval: rearing to the visual $\operatorname{CS}[\operatorname{ts}(5) \geqslant 3.04$, ps $<.05]$, and head-jerk $[\operatorname{ts}(5) \geqslant 2.62$, ps $<.05]$ and startle [Wilcoxon $\mathrm{T}=0, \mathrm{p}<.05$ ] to the noise CS. Behaviors which were dependent on US type occurred more frequently during the latter half of the CS interval: magazine $[\mathrm{t}(5)=3.98, \mathrm{p}<.01]$ and $\operatorname{cup}[\mathrm{ts}(5) \geqslant 3.71$, ps $<.01]$ behavior to the light $\mathrm{CS}$, and magazine $[\mathrm{t}(5)=3.22, \mathrm{p}<.05]$ and cup $[\mathrm{ts} \geqslant 2.88, \mathrm{ps}<.05]$ behavior to the noise CS.

Comparison of CS- and US-evoked responding. Behavior evoked by US delivery and that evoked by CS presentation was similar in some respects but different in others. Cup or magazine behavior was evoked both by US delivery and by CS presentation. Startle responding was evoked by US delivery and by presentation of auditory, but not visual, CSs. Grooming was evoked by sucrose delivery (Groups Lsc and Nsc), but not by either the noise or light CSs paired with sucrose. Rearing was evoked by visual CSs, but not auditory CSs, or US delivery. Head jerk was evoked by auditory CSs, but not visual CSs or US delivery.

\section{Discussion}

The use of qualitatively different USs resulted in somewhat different patterns of conditioned behavior to both auditory and visual CSs. The occurrence of magazine or cup behavior to a CS depended on whether the US used evoked that behavior. But "CS-specific" behaviors-startle and head jerk to the noise and rear to the light-were unaffected by US type. These results support Holland's (1977) hypothesis that rear, startle, and head-jerk behaviors are the result of enhancement of the original $O R$ to the CS, but that the form of other conditioned behaviors such as cup or magazine is determined by the nature of the US.

Interpretation of the insensitivity of startle responding to the noise CS to variation in US type is complicated by the fact that all three USs evoked startle responding. As mentioned earlier, it is likely that startle responding to US delivery was, in fact, conditioned responding to the audible feeder click.

\section{EXPERIMENT 2}

Experiment 2 examined the effects of quantitative variations in the US on various components of conditioned behavior. Separate groups of rats received

Table 2

Temporal Distribution of CS Behavior in Experiment 1

\begin{tabular}{|c|c|c|c|c|c|c|c|}
\hline \multirow[b]{2}{*}{ Behavior } & \multicolumn{3}{|c|}{ Light Groups } & \multirow[b]{2}{*}{ Behavior } & \multicolumn{3}{|c|}{ Noise Groups } \\
\hline & Lfm & Lfc & Lsc & & $\mathrm{Nfm}$ & $\mathrm{Nfc}$ & Nsc \\
\hline Rear & 75.27 & $78-24$ & $73-28$ & Startle & $67-00$ & $66-00$ & $65-00$ \\
\hline Magazine & $12-54$ & & & Head-Jerk & $59-39$ & $61-40$ & $59-42$ \\
\hline \multirow[t]{2}{*}{ Cup } & & $9-53$ & $15-48$ & Magazine & $11-31$ & & \\
\hline & & & & Cup & & $11-31$ & $14-29$ \\
\hline
\end{tabular}

Note-The first number of each pair signifies mean percentage of total behavior during the first 5-sec period of the CS interval; the second number signifies the mean percentage total behavior during the second 5-sec period of the 10-sec CS interval. Startle data signify mean percentage trials on which a startle occurred in the first and second 5-sec periods. 
pairings of a tone or a light CS with one of four magnitudes of food-pellet US. "US-determined" (i.e., magazine) behavior might be expected to be more influential in determining the overall response to the CS with larger magnitude USs than with smaller. Hence, higher frequencies of magazine behavior might be expected when larger magnitude USs are used. Increased magazine behavior might compete with "CS determined" rearing or startle/ head-jerk behaviors; hence, lower frequencies of those behaviors might be anticipated when larger magnitude USs are used.

\section{Method}

Subjects and Apparatus. The subjects were 48 male SpragueDawley rats about 100 days old at the beginning of the experiment. They were maintained at $80 \%$ of their ad-lib weights throughout the experiment. The apparatus was that used in Experiment 1, except that the food cups were removed.

Procedure. The first session served as a pretest of the stimuli to be used in conditioning (rats did not receive separate magazine training in this experiment). Each rat received four 10 -sec presentations of a $75-\mathrm{dB} 1,400-\mathrm{Hz}$ tone and four $10-\mathrm{sec}$ presentations of the light CS. This and all subsequent sessions were $75 \mathrm{~min}$ in duration; no stimuli were delivered during the first $15 \mathrm{~min}$ of any session. Intertrial intervals averaged $7.4 \mathrm{~min}$ throughout the experiment.

Rats were then assigned to one of eight groups of six rats each. In each session of conditioning, rats in Group $\mathrm{T} 1$ received four 10 -sec presentations of the tone CS followed by 1 food pellet; rats in Group T5 received those presentations paired with delivery of 5 pellets; Group T10, 10 pellets; Group T20, 20 pellets. In addition, rats in each of the above groups received four 10-sec nonreinforced presentations of the light CS in each session. Rats in Group $\mathrm{L} 1$ received four 10 -sec presentations of the light CS paired with 1 food pellet in each session; rats in Group LS received those presentations paired with 5 pellets; Group L10, 10 pellets; Group L20, 20 pellets. Rats in each of the latter four groups also received four nonreinforced 10 -sec presentations of the tone CS per session. In all groups, food pellets were delivered to the recessed food magazine at the rate of $4 / \mathrm{sec}$. Conditioning continued for 12 sessions.

\section{Results and Discussion}

Pre-CS behavior. About $90 \%$ of the total pre-CS behavior was quiet. The remaining behavior consisted of rear and magazine. There were no differences among the groups in the frequency of any behavior during the pre-CS periods.

Tone CS behavior. The three left panels of Figure 2 show behavior to the tone CS in groups which received tone-food pairings. For the sake of clarity, behavior to the nonreinforced tone in the remaining groups is not shown. As in Experiment 1, none of the behaviors reported comprised more than $5 \%$ of the total behavior to nonreinforced tones. Contingency (paired-unpaired) by US Magnitude by Sessions analyses of variance indicated that the occurrence of each of these behaviors depended on tone-food pairings $[$ Fs $(1,40) \geqslant 89.11$, ps $<.01]$.

The frequency of startle responding (upper left panel of Figure 2) was not affected by US magnitude
$[\mathrm{F}(3,40)=1.10, \mathrm{p}>.10]$. Although Figure 2 suggests that startle was acquired more rapidly when the tone was paired with larger magnitude, this impression was not supported statistically: the US Magnitude by Sessions interaction was insignificant $[F(33,440)=1.21, p>.10]$. Head-jerk behavior (left-center panel of Figure 2) was affected by US magnitude $[\mathrm{F}(3,40)=2.97, \mathrm{p}<.05]$. An analysis of linear trend showed head jerk to be more frequent when the tone was paired with smaller magnitude USs $[F(1,40)=4.28, p<.05]$. However, a significant US Magnitude by Sessions interaction $[F(33,40)$ $=2.19, \mathrm{p}<.01]$ suggested that, although higher asymptotic frequency of head jerk occurred with smaller magnitude food USs, acquisition of head jerk was more rapid with large-magnitude USs. Magazine behavior (lower left panel of Figure 2) was also affected by US magnitude $[F(3,40)=21.33$, $\mathrm{p}<.01$ ], being more frequent when the tone $\mathrm{CS}$ was paired with larger magnitude USs $[F(1,40)=19.13$, $\mathrm{p}<.01]$.

Light $\mathrm{CS}$ behavior. The upper right panel of Figure 2 shows rearing to the light stimulus in all eight groups. Connected open symbols signify rearing to
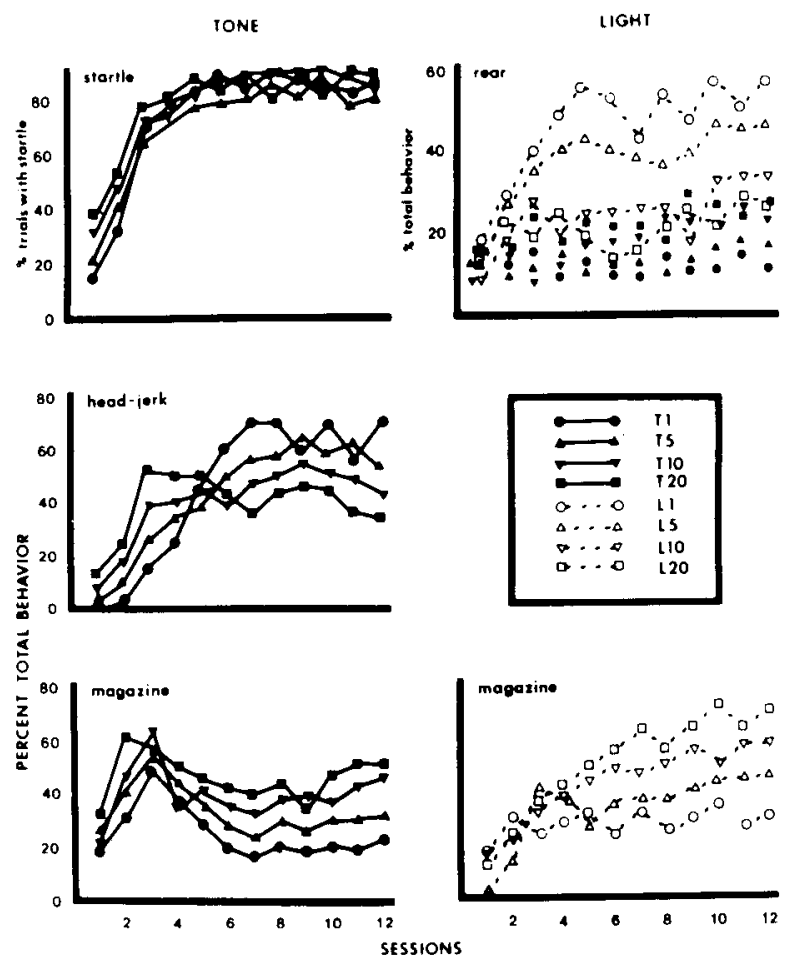

Figure 2. Behaviors to conditioned tone and light stimuli during conditioning in Experiment 2. Frequency of each behavior, except startle, is expressed as a percentage of total behavior; startle behavior is expressed as the percentage of trials on which a startle occurred. Note that the ordinate scale for rearing to the light (upper-right panel) is expanded relative to the scales of the other panels. Note also that rearing to unconditioned light stimuli is also shown in that panel (see text). 
the light in the four groups in which it was paired with food; unconnected solid symbols signify rearing to the light in the groups in which it was unpaired with food. The frequency of rearing was affected by both light-food pairing $[F(1,40)=8.60, p<.01]$ and US magnitude $[F(3,40)=3.22, p<.05]$. However, the effects of US magnitude depended on whether the light was paired with food or not [Contingency by US Magnitude, $F(3,40)=27.22, p<.01]$. Analyses of linear trends among the simple main effects verified the impression that larger magnitude USs resulted in less frequent rearing to reinforced light CSs $[F(1,40)=8.04, p<.01]$ but more frequent rearing to nonreinforced light $\operatorname{CSs}[F(1,40)=4.84$, $\mathrm{p}<.05]$.

The lower right panel of Figure 2 shows magazine behavior to the light $\mathrm{CS}$ in groups which received light-food pairings. For the sake of clarity, behavior to the nonreinforced light in the remaining groups is not shown. As in Experiment 1, magazine behavior comprised less than $5 \%$ of the total behavior to nonreinforced lights. Magazine behavior was more frequent when larger magnitude USs were used $[F(1,40)$ $=9.16, \mathrm{p}<.01]$.

In summary, "US-determined" magazine behavior constituted a larger portion of conditioned behavior to both tone and light CSs when those CSs were paired with larger magnitude USs. Greater frequencies of magazine behavior occurred at the expense of "CS-determined" rearing to the light CS and headjerk behavior to the tone CS. CS-determined startle responding to the tone was unaffected by US magnitude, perhaps because, since it occurred so early in the CS interval, it was not susceptible to competition from increased magazine behavior.

\section{EXPERIMENT 3}

Experiments 1 and 2 showed that both qualitative and quantitative variations in appetitive USs were reflected more directly in late-CS behaviors than in CS-specific, early-CS behaviors. Experiment 3 examined whether similar effects occur in an aversive conditioning situation as well. Tone and light CSs were paired with various shock USs; early-CS, ORbased behaviors (rearing and startle) were expected to resemble those found in Experiments 1 and 2, while late-CS behaviors should be appropriate to the shock US.

Whether pairing a CS with an aversive US maintains or enhances ORs has been a matter of controversy. Sokolov (1963) claimed that they could be so maintained, but other investigators (e.g., Razran, 1930) concluded that ORs habituate rapidly despite pairings with an aversive US. More recently, Shettleworth (1978), studying conditioned behavior of hamsters in a situation similar to that of the present experi- ments, found some evidence of conditioning-specific maintenance of an OR to a tone CS in one experiment but none in a very similar experiment. Rescorla and Holland (1977), using the same conditioning preparation as used here, found a small degree of persistence of a startle response to an auditory CS paired with a shock US. Unfortunately, it is difficult to evaluate Rescorla and Holland's results, since the auditory CS had been previously paired with food and there were no appropriate control groups to allow any decision about the necessity of CS-US pairing in maintaining the response.

It is possible that many failures to see a maintenance of an OR when CSs are paired with aversive USs are due to the OR being masked by very substantial US-generated behaviors. In Experiment 2, large-magnitude appetitive rewards masked OR-based behaviors to a larger extent than did small-magnitude USs; perhaps the aversive USs generally used produce such potent "US-determined" behaviors that any tendency towards maintenance of the $O R$ is masked. In an effort to evaluate this possibility, in Experiment 3 tone or light CSs were paired with one of four shock intensities. Shock intensities great enough to support conditioning yet weak enough not to generate behaviors which completely mask display of the OR might be expected to support more rearing and startle/head-jerk behaviors than the relatively intense shocks often used as USs.

\section{Method}

Subjects and Apparatus. The subjects were 48 male SpragueDawley rats about 100 days old at the beginning of the experiment. They were maintained at their ad-lib weights throughout the experiment. The apparatus was that used in Experiment 2.

Behavioral observation. Behavioral observation procedures were identical to those of Experiments 1 and 2, except that another behavior, freeze, a characteristic motionless crouching behavior recorded previously by Rescorla and Holland (1977), was recorded.

Procedure. The first four sessions served as a pretest of the stimuli to be used in conditioning. In each session, rats received two $10-\mathrm{sec}$ presentations of the $1,400-\mathrm{Hz}$ tone and two $10-\mathrm{sec}$ presentations of the light CS. These and all subsequent sessions were $75 \mathrm{~min}$ in duration; no stimuli were delivered during the first 15 min of any session.

Rats were then randomly assigned to one of eight groups of six rats each, and treatment designed to establish conditioning to either the light or the tone was begun. In each conditioning session, rats in Groups T10, T25, T35, and T50 received two pairings of the 10-sec tone with a .5-sec grid shock of $.10, .25$, .35 , or $.50 \mathrm{~mA}$, respectively, and two nonreinforced presentations of the 10-sec light stimulus. Groups L10, L25, L35, and L50 received two pairings of the 10-sec light CS with a .5 -sec shock of $.10, .25, .35$, or $.50 \mathrm{~mA}$, respectively, and two nonreinforced presentations of the $10-\mathrm{sec}$ tone stimulus. There were six conditioning sessions.

\section{Results and Discussion}

Pre-CS behavior. Pre-CS behavior consisted of quiet and freeze behaviors. Table 3 shows the frequency of pre-CS freeze behaviors in the eight groups 
Table 3

Pre-CS Freeze Behavior in Experiment 3

\begin{tabular}{cccccccc}
\hline T10 & T25 & T35 & T50 & L10 & L25 & L35 & L50 \\
\hline 0 & 12 & 19 & 26 & 2 & 13 & 17 & 23 \\
\hline
\end{tabular}

Note-Data represent freeze behavior in each of eight groups over all conditioning sessions, expressed as percentages of total pre-CS behavior.

over all conditioning sessions. Pre-CS freeze behavior differed as a function of shock intensity $[F(3,40)$ $=3.04, \mathrm{p}<.05]$ but was not affected by the type of CS paired with shock $[\mathrm{F}(1,40)<1]$.

Behavior to tone CS. The two left frames of Figure 3 show behavior evoked by the tone stimulus in all eight groups over the course of conditioning. The frequency of startle responding (upper left) was affected by both tone-shock contingency (paired or unpaired) $[F(1,40)=8.41, p<.01]$ and shock intensity $[F(3,40)=7.38, p<.01]$. Individual comparisons using the Scheffé procedure indicated that only groups T25 and T35 differed from their corresponding controls, Groups L25 and L35 $[\mathrm{Fs}(7,40) \geqslant 16.73$, ps $<.05]$. Startle was maintained at higher levels in the two intermediate intensity groups considered together than in low (T10) and high (T50) intensity groups $[F(7,40)=16.90, p<.05]$.

The frequency of freezing to the tone CS (lower left) was also affected by both tone-shock contingency $[F(1,40)=13.11, p<.01]$ and shock intensity $[\mathrm{F}(3,40)=11.78, \mathrm{p}<.01]$. An analysis of linear
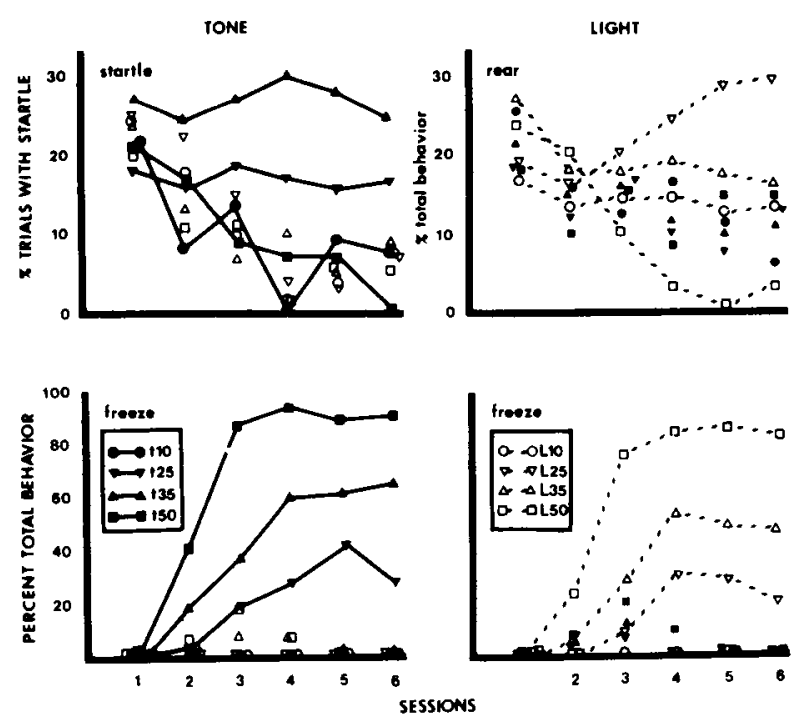

Figure 3. Behaviors to tone and light CSs during conditioning in Experiment 3. Frequency of rear and freeze is expressed as a percentage of total behavior; startle responding is expressed as a percentage of trials on which a startle response occurred. Note that the ordinate scale for rear and freeze differ. Connected symbols signify behaviors to CSs paired with shock: unconnected symbols signify behaviors to CSs unpaired with shock. trend among the four groups receiving tone-shock pairings showed that higher shock intensities produced higher levels of freezing $[\dot{F}(1,40)=14.42$, $\mathrm{p}<.01]$.

Behavior to light CS. The two right panels of Figure 3 show behavior evoked by the light stimulus in all eight groups over the course of conditioning. The frequency of rearing (upper right) was affected by both light-shock contingency $[F(1,40)=6.88$, $\mathrm{p}<.05]$ and shock intensity $[\mathrm{F}(3,40)=7.17$, $\mathrm{p}<.01]$. Individual Scheffé comparisons indicated that only Groups L25 and L35 differed from their corresponding control groups $[\mathrm{Fs}(7,40) \geqslant 16.00$, ps $<.05$ ]. Rearing was maintained at higher levels in the two intermediate-intensity groups together than in low- or high-intensity groups $[F(7,40)=15.95$, $\mathrm{p}<.05]$.

Freezing to the light CS (lower right) was also affected by both light-shock contingency $[F(1,40)=$ $12.84, p<.01]$ and shock intensity $[F(3,40)=10.99$, $\mathrm{p}<.01]$. An analysis of linear trend among the groups receiving light-shock pairings showed that higher intensity shocks produced higher levels of freezing $[F(1,40)=14.81, p<.01]$.

Thus, the results of Experiment 3 paralleled those of Experiment 2, in which an appetitive US was used. Higher intensity USs resulted in more frequent USdependent freezing to both tone and light CSs, but less frequent CS-dependent rearing or startle behaviors. The conditioning-dependent maintenance of rearing and startle when CSs were paired with intermediate intensity shocks supports claims that ORs can be maintained by pairing with aversive USs, and indicates that the frequent failure to find such maintenance may be the result of masking of the OR by competing, US-generated conditioned behaviors.

\section{GENERAL DISCUSSION}

Individual components of conditioned behavior were differentially affected by both qualitative and quantitative variations in the US. In Experiment 1, conditioned behavior occurring more frequently during the later portion of the CS-US interval resembled responding to the qualitatively different appetitive USs used. But behaviors occurring more frequently during the early portions of the CS-US interval (rearing to visual CSs or startle and head jerk to auditory CSs) were unaffected by the nature of the US. In Experiment 2, larger magnitude food USs supported higher frequencies of conditioned behavior resembling the response to the US, but lower frequencies of rearing, startle, and head-jerk behaviors. In Experiment 3, higher intensity shock USs supported higher levels of freezing behavior appropriate to shock USs but lower levels of rearing and startle behaviors. 
These data are consistent with Holland's (1977) orienting response hypothesis. According to that hypothesis, CS-US contingencies are thought to result in two quite independent types of conditioned behavior to the CS-a response appropriate to the US, perhaps as a consequence of a stimulus substitution process (e.g., Mackintosh, 1974), and enhancement of the OR originally elicited by the CS, perhaps via increases in attention to the CS (e.g., Sokolov, 1963; Sutherland \& Mackintosh, 1971), or sensitization of the CS-OR reflex (Dykman, 1967).

Both the temporal distribution of various behaviors and their sensitivity to US variations found in these experiments are anticipated within this hypothesis. Since ORs occur most often near stimulus onset, OR-based components of the CRs would be expected to occur most frequently during early portions of CSs. Similarly, since the US is in closer proximity to later portions of the CS, US-dependent components of the CRs would be expected to be most frequent during later portions of CSs. Consequently, the direct effects of qualitative and quantitative manipulations of the US would be expected to be confined to late-CS, US-determined behaviors, as was found in these experiments. Conversely, the effects of manipulations of CS characteristics would be expected to be confined to early-CS, OR-based responding; this expectation has been confirmed elsewhere (Holland, in press).

Some evidence suggests that the individual component behaviors observed here may not be determined solely by CS or solely by US characteristics. For instance, although the results of Experiments 1 and 2 indicated that head-jerk behavior to auditory CSs was CS rather than US dependent, the complete absence of that behavior when the tone was paired with shock USs in Experiment 3 may indicate that head-jerk behavior is determined to some extent by the nature of the US. Similarly, other experiments (e.g., Rescorla, 1978) indicate that a rat's body orientation when exhibiting head-jerk behavior depends on the location of the reinforcement delivery cup. Perhaps, as Holland (1977) suggested, head jerk is the result of interaction between US-dependent behaviors (e.g., magazine and cup) and the aftereffects of startle responding. Thus, the origins of various components of conditioned behavior might be better described as falling on a continuum ranging from unique determination by the US, through joint determination by US and CS, to CS-specific behaviors.

Other processes besides those specified by the OR hypothesis may influence the form of appetitive conditioned responses. For example, Jenkins, Berrera, Ireland, and Woodside (1978) proposed that behaviors occurring to CS-US sequences in conditioning experiments mimic those occurring during naturally occurring episodes. The CS thus substitutes, not for the US, but for some "natural signal" for food. Various CSs might substitute for different natural signals, resulting in apparently CS-specific behavior appropriate to those natural signals. For example, Jenkins et al. (1978) described dogs' behaviors in the presence of auditory-visual CSs paired with food as representing hunting/soliciting behaviors naturally occurring prior to access to food. But this account lacks specificity; in most cases it is not clear how it may be used to predict the form of behavior occurring to various conditioned signals for food. The orienting response hypothesis presented above provides considerable specificity; many detailed features of conditioned responding in the conditioning situation used here are predictable from features of initially unconditioned orienting responses to the CS (Holland, 1977, in press).

Another alternative to the OR hypothesis is that only late-CS, US-dependent components of conditioned behavior are true Pavlovian CRs, and that early-CS behaviors such as startle and rearing are the result of instrumental contingencies. That is, delivery of a food US just after the occurrence of initially unconditioned startle or rearing may have resulted in the establishment of those behaviors as discriminative operants. However, considerable data make instrumental origin of these behaviors seem unlikely. First, startle and rear responses developed even when considerable care was taken to insure that initially unconditioned ORs were not adventitiously paired with food. Holland (1977) found acquisition of these behaviors even after they were extensively habituated before food reinforcement was introduced. Further, Holland (1979) showed that startle and rearing showed only minimal sensitivity to omission contingencies. That is, substantial acquisition of those behaviors occurred even when US presentation was contingent on their nonoccurrence. Second, it is unlikely that shock delivery served as a positive instrumental reinforcer in Experiment 3, yet startle and rearing were maintained. Hence, CS-determined rearing and startle behaviors seem to be supported by CS-US contingencies (as assumed in the OR hypothesis) rather than instrumental, responsereinforcer contingencies.

Data such as these showing that production of conditioned responding to Pavlovian CSs is not a unitary process but involves at least two somewhat independent sources of responding suggests that care must be taken in making inferences about processes of association or "cognitive processing" from many measures of conditioned responding. Various components of conditioned behavior may be differentially affected by particular manipulations, as in these experiments. Indeed, some investigators (e.g., Maltzman, 1977) have proposed that conditioning of ORs, for example, may be different from other types 
of conditioning. Depending on what components of conditioned behavior affect a chosen response measure most heavily, one might make quite different statements about underlying learning processes from the results of conditioning experiments.

\section{REFERENCES}

Dyкman, R. A. On the nature of classical conditioning. In C. C. Brown (Ed.), Methods in psychophysiology. Baltimore: Williams \& Wilkins, 1967.

Hilgard, E. G., \& Marquis, D. G. Conditioning and learning. New York: Appleton-Century, 1940.

Holland, P. C. Conditioned stimulus as a determinant of the form of the Pavlovian conditioned response. Journal of Experimental Psychology: Animal Behavior Processes, 1977, 3, 77-104.

Holland, P. C. Differential effects of omission contingencies on various components of Pavlovian appetitive conditioned behavior in rats. Journal of Experimental Psychology: Animal Behavior Processes, 1979, 5, 178-193.

Holland, P. C. Influence of visual CS characteristics on the form of Pavlovian appetitive conditioned behavior in rats. Journal of Experimental Psychology: Animal Behavior Processes, in press.

Jenkins, H. M., Berrera, F. J., Ireland, C., \& Woodside, B. Signal-centered action patterns of dogs in appetitive classical conditioning. Learning and Motivation, 1978, 9, 272-296.
Jenkins, H. M., \& Moone, B. R. The form of the autoshaped response with food or water reinforcers. Journal of the Experimental Analysis of Behavior, 1973, 20, 163-181.

Mackintosh, N. J. The psychology of animal learning. New York: Academic Press, 1974.

Maltzman, I. Orienting in classical conditioning and generalization of the galvanic skin response to words: An overview. Journal of Experimental Psychology: General, 1977, 106, 111-119.

RAzran, G. H. S. Theory of conditioning and related phenomena. Psychological Review, 1930, 37, 25-43.

Resconla, R. A. Some implications of a cognitive approach to conditioning. In S. H. Hulse, H. Fowler, \& W. K. Honig (Eds.), Cognitive processes in animal behavior. Hillsdale, N.J: Erlbaum, 1978.

Rescorla, R. A., \& Holland, P. C. Associations in Pavlovian conditioned inhibition. Learning \& Motivation, 1977, 8, 429-447.

Rescorla, R. A., \& Solomon, R. L. Two process learning theory: Relationships between classical conditioning and instrumental learning. Psychological Review, 1967, 74, 151-182.

ShetTleworth, S. J. Reinforcement and the organization of behavior in golden hamsters: Pavlovian conditioning with food and shock USs. Journal of Experimental Psychology: Animal Behavior Processes, 1978, 4, 152-169.

Sokolov, Y. N. Perception and the conditioned reflex. Oxford: Pergamon, 1963.

Sutherland, N. S., \& Mackintosh, N. J. Mechanisms of animal discrimination learning. New York: Academic Press, 1971.

(Received for publication December 29, 1978; revision accepted April 18, 1979.) 